\title{
SYNTHESIS AND BIOLOGICAL ACTIVITY OF ANALOGUES \\ OF DIAZAQUINOMYCIN A, A NEW THYMIDYLATE SYNTHASE INHIBITOR
}

\author{
Kazuo Tsuzuki, Tadao Yokozuka, Masatsune Murata, ${ }^{\dagger}$ \\ Haruo Tanaka and Satoshi ÖMURA \\ The Kitasato Institute, and School of Pharmaceutical Sciences, Kitasato University, \\ Minato-ku, Tokyo 108, Japan
}

(Received for publications September 2, 1988)

\begin{abstract}
Diazaquinomycin A (1), a new thymidylate (TMP) synthase inhibitor, is poorly soluble in various solvents and exhibits no antitumor activity, while a series of the analogues prepared from 1 are more soluble in water and chloroform than 1 , and some of them exhibit antitumor activity in mice. Some analogues in which the lactam rings are replaced by pyridine rings did not inhibit TMP synthase. The diethoxy analogue 25 is a 10 -fold more potent inhibitor of TMP synthase than 1 . The diacetoxy analogue 23 exhibits significant antitumor activity (T/C: $175 \%$ ) against Meth-A fibrosarcoma in mice.
\end{abstract}

Diazaquinomycin A (1), possessing an unique 1,8-diazaanthraquinone skeleton, is a new antibiotic found as a folic acid antagonist by a screening method established for inhibitors of folate metabolism. ${ }^{1 \sim 4)}$ Recently, some of the present authors have shown that 1 inhibits thymidylate (TMP) synthase competitively with 5,10-methylenetetrahydrofolate and exhibits effective cytotoxicity against tumor cells, but has no in vivo effect on tumors in mice, probably because of its extremely low solubility. In fact, solid residues of 1 were observed when mice were dissected after a suspension of 1 in $0.5 \%$ gum arabic was administered intraperitoneally.

This paper describes the synthesis of diazaquinomycin analogues with the aim to improve the solubility of 1 and to evaluate their in vitro and in vivo activities, including antitumor activity.

Chemical modification of $\mathbf{1}$ is limited by its very low solubility in organic and aqueous media. However, conversion of the lactam rings of $\mathbf{1}$, which are necessary for manifestation of biological activity, to 2-methoxypyridine rings improved the solubility in organic solvents and served to activate the methyl groups, which could then be brominated. Nucleophilic substitution of the aryl bromide by several nucleophiles provided a versatile method to introduce polar groups onto the methyl groups of 1 . Thereafter, acidic treatment reconstituted the 2-oxopyridine rings.

\section{Chemistry}

1 was treated with methyl iodide and silver oxide in chloroform to give the imino ether 2 possessing one 2-methoxypyridine and one lactam ring. Refluxing the reaction mixture afforded 3. Bromination of the two imino ethers gave 4 and 5. Substituting the bromides by a series of nucleophiles such as cyanide, malonate anion, water, acetic acid, ethanol and morpholine, and then hydrolyzing the imino ethers with $\mathrm{H}_{2} \mathrm{SO}_{4}$ - EtOH gave the diazaquinomycin analogues as shown in Scheme 1.

t Present address: Department of Nutrition and Food Science, Ochanomizu University, Otsuka, Bunkyoku, Tokyo 112, Japan. 
Scheme 1.<smiles>CCCc1c2c([nH]c(=O)c1C)C(=O)c1[nH]c(=O)c(C)c(CCC)c1C2=O</smiles>

1<smiles>CCCc1c(C)c(OC)nc2c1C(=O)c1c([nH]c(=O)c(C)c1CCC)C2=O</smiles>

2<smiles>CCCc1c(C)c(OC)nc2c1C(=O)c1c(nc(OC)c(C)c1CCC)C2=O</smiles>

3<smiles>CCCc1c(C)c(C)nc(OC)c1C</smiles>

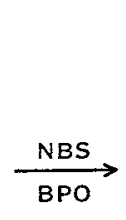<smiles>CCCc1c(C)c(C)nc(OC)c1CBr</smiles>

4 and 5

NBS: $N$-Bromosuccinimide

BPO: Benzoyl peroxide

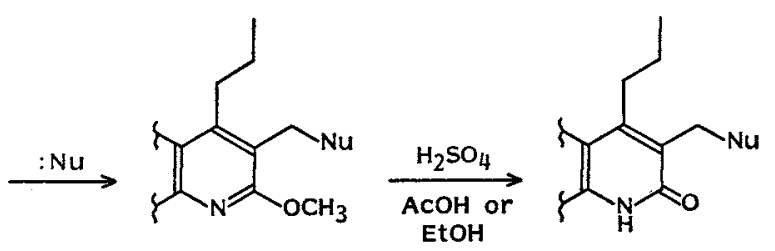

20 and $22 \mathrm{Nu}=\mathrm{OAc}$

21 and $23 \mathrm{Nu}=\mathrm{OAc}$

26<smiles>N#CN1CCOCC1</smiles>

24 and $25 \mathrm{Nu}=\mathrm{OEt}$

27<smiles>N#CN1CCOCC1</smiles>

Scheme 2.<smiles>CCCc1c(CBr)c(OC)nc2c1C(=O)c1c(nc(OC)c(CBr)c1CCC)C2=O</smiles>

5<smiles>[R]c1c(OC)nc2c(=O)c3c(c(CCC)c1[R])c(=O)c1c(CCC)c(OC)nc2c13</smiles>

6 $\mathrm{R}=\mathrm{CH}_{2} \mathrm{CN}$

$9 \mathrm{R}=\mathrm{CH}_{2} \mathrm{CH}(\mathrm{COOEt})_{2}$

$12 \mathrm{R}=\mathrm{CH}_{2} \mathrm{CH}(\mathrm{COOH})_{2}$

$13 \mathrm{R}=\mathrm{CH}_{2} \mathrm{CH}_{2} \mathrm{COOH}$

$16 \mathrm{R}=\mathrm{CH}_{2} \mathrm{OH}$

$17 \mathrm{R}=\mathrm{COOH}$ $\underset{\text { EtOH }}{\stackrel{\mathrm{H}_{2} \mathrm{SO}_{4}}{\longrightarrow}}$<smiles></smiles>

$7 \mathrm{R}=\mathrm{CH}_{2} \mathrm{COOEt}$

$8 \mathrm{R}=\mathrm{CH}_{2} \mathrm{COOH}$

$10 \mathrm{R}=\mathrm{CH}_{2} \mathrm{CH}(\mathrm{COOEt})_{2}$

$11 \mathrm{R}=\mathrm{CH}_{2} \mathrm{CH}(\mathrm{COOH})_{2}$

$14 \mathrm{R}=\mathrm{CH}_{2} \mathrm{CH}_{2} \mathrm{COOEt}$

$15 \mathrm{R}=\mathrm{CH}_{2} \mathrm{CH}_{2} \mathrm{COOH}$

$18 \mathrm{R}=\mathrm{COOEt}$

$19 \mathrm{R}=\mathrm{COOH}$

Cyanation of the dibromide 5 with tetraethylammonium cyanide ${ }^{5)}$ gave the dicyanide $6 .{ }^{8)}$ The cyano groups were converted to ethyl esters with $\mathrm{H}_{2} \mathrm{SO}_{4}-\mathrm{EtOH}$ and at the same time the imino ethers were hydrolyzed to give the ester 7. Substitution of 5 by ethyl malonate anion gave the ester $\mathbf{9}$, and subsequent hydrolysis with $\mathrm{H}_{2} \mathrm{SO}_{4}$ - EtOH gave the dimalonate ester 10. Successive treatments of 9, such as hydrolysis of the esters with $\mathrm{NaOH}-$ aq $\mathrm{EtOH}$, decarboxylation in pyridine and hydrolysis of the imino ethers with $\mathrm{H}_{2} \mathrm{SO}_{4}-\mathrm{EtOH}$, gave the ester 14. Hydrolysis of the dibromide 5 with water and THF at reflux without acid and base gave the diol 16. The hydroxyl groups were oxidized with $\mathrm{CrO}_{3}-\mathrm{H}_{2} \mathrm{SO}_{4}$ leading to the dicarboxylic acid 17. Further treatment of 17 with $\mathrm{H}_{2} \mathrm{SO}_{4}-\mathrm{EtOH}$ afforded the ester 18. Hydrolysis with $\mathrm{NaOH}$ - aq EtOH gave the corresponding carboxylic acids as shown in Scheme 2. 
Table 1. In vitro activities and solubilities of diazaquinomycin $\mathrm{A}$ analogues.

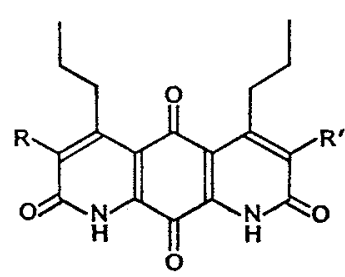

Type 1

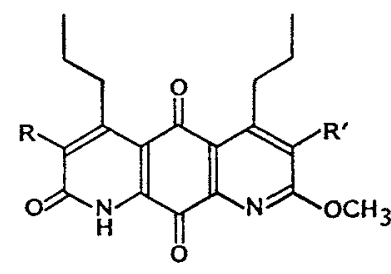

Type II

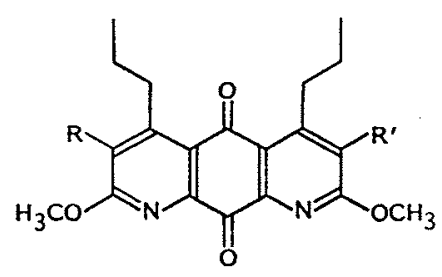

Type III

\begin{tabular}{|c|c|c|c|c|c|c|c|c|c|}
\hline \multirow{3}{*}{ Compound } & \multirow{3}{*}{ Type } & \multirow{3}{*}{$\mathrm{R}$} & \multirow{3}{*}{$\mathrm{R}^{\prime}$} & \multicolumn{4}{|c|}{ Inhibitory activity against } & \multirow{2}{*}{\multicolumn{2}{|c|}{$\begin{array}{l}\text { Solubility } \\
(\mu \mathrm{g} / \mathrm{ml})\end{array}$}} \\
\hline & & & & \multicolumn{2}{|c|}{ Enterococcus faecium } & \multirow{2}{*}{$\begin{array}{c}\text { HeLa } \\
\text { cells } \\
\text { MIC } \\
(\mu \mathrm{g} / \mathrm{ml})\end{array}$} & \multirow{2}{*}{$\begin{array}{c}\text { TMP } \\
\text { synthase }^{\mathrm{a}} \\
\mathrm{IC}_{50} \\
(\mu \mathrm{g} / \mathrm{ml})\end{array}$} & & \\
\hline & & & & $\begin{array}{c}\text { MIC } \\
(\mu \mathrm{g} / \mathrm{ml})\end{array}$ & $\begin{array}{c}\text { Reversal } \\
\text { with 'TdR }\end{array}$ & & & $\mathrm{CHCl}_{3}$ & $\mathrm{H}_{2} \mathrm{O}$ \\
\hline 1 & I & $\mathrm{CH}_{3}$ & $\mathrm{CH}_{3}$ & $1.0 \sim 2.0$ & ++ & $0.16 \sim 0.31$ & 4.2 & 640 & 0.15 \\
\hline 2 & II & $\mathrm{CH}_{3}$ & $\mathrm{CH}_{3}$ & $>500$ & & $>5$ & $>20$ & & \\
\hline 3 & III & $\mathrm{CH}_{3}$ & $\mathrm{CH}_{3}$ & $>500$ & & 2.5 & $>20$ & & \\
\hline 6 & III & $\mathrm{CH}_{2} \mathrm{CN}$ & $\mathrm{CH}_{2} \mathrm{CN}$ & $>500$ & & 1.25 & $>20$ & & \\
\hline 7 & I & $\mathrm{CH}_{2} \mathrm{COOEt}$ & $\mathrm{CH}_{2} \mathrm{COOEt}$ & 3.9 & + & 0.31 & 1.1 & 5,980 & 1.8 \\
\hline 8 & I & $\mathrm{CH}_{2} \mathrm{COOH}$ & $\mathrm{CH}_{2} \mathrm{COOH}$ & $>500$ & & $>5$ & 1.5 & & \\
\hline 10 & I & $\mathrm{CH}_{2} \mathrm{CH}(\mathrm{COOEt})_{2}$ & $\mathrm{CH}_{2} \mathrm{CH}(\mathrm{COOEt})_{2}$ & 62.5 & + & 2.5 & 1.6 & 4,360 & 0.62 \\
\hline 11 & I & $\mathrm{CH}_{2} \mathrm{CH}(\mathrm{COOH})_{2}$ & $\mathrm{CH}_{2} \mathrm{CH}(\mathrm{COOH})_{2}$ & $>500$ & & 5 & 1.3 & & \\
\hline 14 & I & $\mathrm{CH}_{2} \mathrm{CH}_{2} \mathrm{COOE} t$ & $\mathrm{CH}_{2} \mathrm{CH}_{2} \mathrm{COOEt}$ & 3.9 & + & 1.25 & 2.1 & & \\
\hline 15 & I & $\mathrm{CH}_{2} \mathrm{CH}_{2} \mathrm{COOH}$ & $\mathrm{CH}_{2} \mathrm{CH}_{2} \mathrm{COOH}$ & $>500$ & & 5 & 3.4 & & \\
\hline 18 & I & COOEt & COOEt & 250 & & 5 & 1.0 & & \\
\hline 19 & I & $\mathrm{COOH}$ & $\mathrm{COOH}$ & $>500$ & & $>5$ & 2.4 & & \\
\hline 21 & I & $\mathrm{CH}_{3}$ & $\mathrm{CH}_{2} \mathrm{OAc}$ & 2.0 & + & 0.63 & 2.0 & & \\
\hline 22 & III & $\mathrm{CH}_{2} \mathrm{OAc}$ & $\mathrm{CH}_{2} \mathrm{OAc}$ & $>500$ & & 2.5 & $>20$ & & \\
\hline 23 & $I$ & $\mathrm{CH}_{2} \mathrm{OAc}$ & $\mathrm{CH}_{2} \mathrm{OAc}$ & 3.9 & \pm & 0.31 & 1.0 & 3,180 & 0.51 \\
\hline 24 & I & $\mathrm{CH}_{3}$ & $\mathrm{CH}_{2} \mathrm{OEt}$ & 1.0 & ++ & 0.31 & 0.7 & & \\
\hline 25 & I & $\mathrm{CH}_{2} \mathrm{OEt}$ & $\mathrm{CH}_{2} \mathrm{OEt}$ & 1.0 & $+t$ & 0.16 & 0.4 & 3,500 & 0.22 \\
\hline 27 & $\mathbf{I}$ & $\mathrm{CH}_{2} \mathrm{~N}$ & $\mathrm{CH}_{2}{ }^{\mathrm{N}}$ & $>500$ & & $>5$ & 1.0 & & \\
\hline
\end{tabular}

a Prepared from Ehrlich ascites carcinoma cells.

++ : Well reversed, $+:$ moderately reversed, $t:$ weakly reversed. 
Treatment of the monobromide 4 and the dibromide 5 with sodium acetate and acetic acid gave the acetates $\mathbf{2 0}$ and $\mathbf{2 2}$, which were converted to the monoacetate $\mathbf{2 1}$ and the diacetate $\mathbf{2 3}$, respectively, with $\mathrm{H}_{2} \mathrm{SO}_{4}-\mathrm{AcOH}$.

Alcoholysis of 4 and 5 with $\mathrm{H}_{2} \mathrm{SO}_{4}$ - EtOH gave the ethyl ethers 24 and 25, respectively, accompanied by hydrolysis of the imino ether. Reaction of $\mathbf{5}$ with morpholine and a following hydrolysis of the imino ether gave the diamine 27.

\section{Results and Discussion}

Table 1 summarizes the antimicrobial activities of the synthesized analogues of 1 against the folate requiring Enterococcus faecium, their reversal by thymidine (TdR), the cytotoxicities against HeLa cells, the inhibitory activities against TMP synthase prepared from Ehrlich ascites carcinoma cells, and the solubilities in water and chloroform.

All mono- or dimethylated analogues of 1 (Types II and III) exhibited extremely reduced biological activities. The carboxylic acid analogues $(8,11,15$ and 19) were devoid of activities against E. faecium and HeLa cells, but the corresponding esters $(7,10,14$ and 18) were active. Both the carboxylic acids and their esters, however, were more potent inhibitors of TMP synthase than 1 . These results suggest that the carboxylic acids cannot penetrate the cell membrane. 18 exhibited poor activity against E. faecium, but $\mathbf{7}$ and $\mathbf{1 4}$ both containing longer side chains exhibited similar activities against $E$. faecium as did 1 . 7 was most cytotoxic.

Compounds 21 and $\mathbf{2 4}$, which have substituents on one of the two methyl groups of $\mathbf{1}$, were only half as potent as $\mathbf{2 3}$ and $\mathbf{2 5}$, which have the corresponding substituents on both methyl groups of $\mathbf{1}$. All analogues containing two lactam rings (Type I) were more potent inhibitors of TMP synthase

Fig. 1. Mode of inhibitory action of 25 against TMP synthase from Ehrlich ascites carcinoma cells.

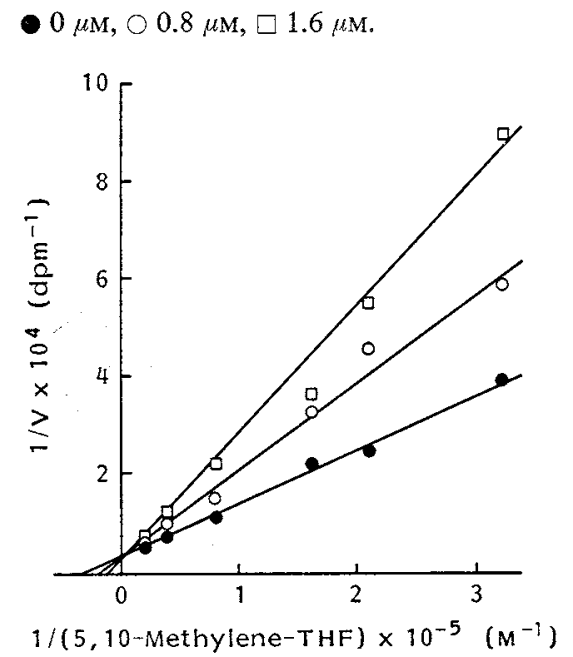

The results of Lineweaver-Burk plot showed that it inhibits the enzyme competitively with the substrate 5,10-methylene-THF. With the enzyme, $K m$ was $45 \mu \mathrm{M}$, and $K i$ was $14 \mu \mathrm{M}$.
Table 2. Antitumor activities of diazaquinomycin A analogues on Meth-A fibrosarcoma.

\begin{tabular}{|c|c|c|c|}
\hline Compound & $\begin{array}{c}\text { Dose } \\
(\mathrm{mg} / \mathrm{kg} / \\
\text { day } \times \text { days })\end{array}$ & $\begin{array}{c}\text { Mean } \\
\text { survival } \\
\text { days }\end{array}$ & $\begin{array}{l}\mathrm{T} / \mathrm{C} \\
(\%)\end{array}$ \\
\hline \multirow[t]{3}{*}{1} & $1 \times 4$ & 12.0 & 96 \\
\hline & $10 \times 4$ & 12.0 & 96 \\
\hline & $100 \times 4$ & 11.5 & 92 \\
\hline \multirow[t]{3}{*}{7} & $1 \times 4$ & 12.3 & 100 \\
\hline & $10 \times 4$ & 12.4 & 101 \\
\hline & $100 \times 4$ & $14.3 \pm 0.75$ & 116 \\
\hline \multirow[t]{3}{*}{23} & $1 \times 4$ & 12.2 & 99 \\
\hline & $10 \times 4$ & $17.3 \pm 2.56$ & $141 *$ \\
\hline & $100 \times 4$ & $21.5 \pm 2.06$ & $175^{*}$ \\
\hline \multirow[t]{3}{*}{25} & $1 \times 4$ & 12.1 & 98 \\
\hline & $10 \times 4$ & 11.8 & 96 \\
\hline & $100 \times 4$ & 12.5 & 102 \\
\hline $5-\mathrm{FU}$ & $20 \times 1$ & 17.5 & 142 \\
\hline Control & - & $12.3 \pm 0.95$ & 100 \\
\hline
\end{tabular}

* $P<0.05$.

5-FU: 5-Fluorouracil. 
than 1. Among them, 25 was the most potent inhibitor of TMP synthase and inhibited the enzyme competitively with 5,10-methylene-THF as substrate (Fig. 1). The inhibitory activity of 25 was 10fold stronger than that of $\mathbf{1}$.

Among the synthesized analogues, three compounds (7, 23 and 25) exhibited activities against HeLa cells similar to those of $\mathbf{1}$, and were more soluble in water and chloroform than $\mathbf{1}$. Their antitumor activities against Meth-A fibrosarcoma in mice were examined and the results are summarized in Table 2. The diacetoxy derivative 23 exhibited significant antitumor activity ( $\mathrm{T} / \mathrm{C}: 175 \%$ ) corresponding to that of 5-fluorouracil (5-FU).

\section{Experimental}

MP's were determined on a micro melting point apparatus (Yanaco MP-3). They were uncorrected. NMR spectra were run in $\mathrm{CDCl}_{3}$ or $\mathrm{MeOH}-d_{4}$ on a $90 \mathrm{MHz}$ spectrometer (Jeol FX-90Q). IR spectra were taken on a Jasco A-102 spectrometer. MS were determined by high-resolution electron impact mass spectrometer (JMS-DX300, JMA-3100). Elemental analysis for carbon, hydrogen and nitrogen were determined with a Perkin-Elmer Model 240 elemental analyzer. Column chromatography was performed on Silica gel 60 (Art. No. 7734, Merck).

2-Methoxy-3,7-dimethyl-4,6-dipropylpyrido[3,2-g]quinoline-5,8,10(9H)-trione (2)

A solution of diazaquinomycin A (1) $(203 \mathrm{mg}, 0.57 \mathrm{mmol})$, methyl iodide $(4 \mathrm{ml}, 64.2 \mathrm{mmol})$ and silver oxide $(398 \mathrm{mg}, 1.72 \mathrm{mmol})$ in $\mathrm{CHCl}_{3}(20 \mathrm{ml})$ was stirred for 20 hours at room temperature. The mixture was filtered and the filtrate was evaporated. The resulting crude product was purified by column chromatography, eluting with $\mathrm{CHCl}_{3}$. An orange crystalline solid was obtained, $151 \mathrm{mg}$ $(72 \%):$ MP $288 \sim 289^{\circ} \mathrm{C}$; NMR Table 3; IR $\left(\mathrm{CHCl}_{3}\right) \mathrm{cm}^{-1} 3350,2960,1645$; MS calcd for $\mathrm{C}_{21} \mathrm{H}_{24} \mathrm{~N}_{2} \mathrm{O}_{4}$ 368.174, observed 368.174 .

\section{2,8-Dimethoxy-3,7-dimethyl-4,6-dipropylpyrido[3,2-g]quinoline-5,10-dione (3)}

A suspension of $1(250 \mathrm{mg}, 0.71 \mathrm{mmol})$, methyl iodide $(5 \mathrm{ml}, 80.3 \mathrm{mmol})$ and silver oxide $(500$ $\mathrm{mg}, 2.16 \mathrm{mmol})$ in $\mathrm{CHCl}_{3}(25 \mathrm{ml})$ was heated at reflux for 2 hours. As described for 2 , the mixture was treated and purified to give a yellow crystalline solid, $235 \mathrm{mg}(87 \%)$ : MP $136 \sim 138^{\circ} \mathrm{C}$; NMR Table 3; IR $\left(\mathrm{CHCl}_{3}\right) \mathrm{cm}^{-1} 2970,2880,1695,1655$; MS calcd for $\mathrm{C}_{22} \mathrm{H}_{26} \mathrm{~N}_{2} \mathrm{O}_{4}, 382.189$, observed 382.189.

3-Bromomethyl-2-methoxy-7-methyl-4,6-dipropylpyrido[3,2-g]quinoline-5,8,10(9H)-trione (4)

A solution of $2(242 \mathrm{mg}, 0.66 \mathrm{mmol}), N$-bromosuccinimide $(242 \mathrm{mg}, 1.36 \mathrm{mmol})$ and benzoyl peroxide $(20 \mathrm{mg}, 0.08 \mathrm{mmol})$ in $\mathrm{CCl}_{4}(93 \mathrm{ml})$ was heated at reflux for 1 hour. The mixture was cooled at $0^{\circ} \mathrm{C}$ and filtered. The solids were suspended in $\mathrm{CCl}_{4}$ and kept at $50^{\circ} \mathrm{C}$ for 30 minutes. The suspension was filtered, and the solids were dissolved in $\mathrm{CHCl}_{3}(100 \mathrm{ml})$. The solution was washed with water $(100 \mathrm{ml})$ and dried over anhydrous $\mathrm{Na}_{2} \mathrm{SO}_{4}$. After filtration the solvent was removed to yield a yellow crystalline solid, $224 \mathrm{mg}(76 \%)$ : MP $248 \sim 250^{\circ} \mathrm{C}$; NMR Table 3; IR $\left(\mathrm{CHCl}_{3}\right) \mathrm{cm}^{-1} 3350$, 2960, 2925, 1655, 1600; MS calcd for $\mathrm{C}_{21} \mathrm{H}_{23} \mathrm{BrN}_{2} \mathrm{O}_{4}, 446.084$, observed 446.082.

\section{3,7-Dibromomethyl-2,8-dimethoxy-4,6-dipropylpyrido[3,2-g]quinoline-5,10-dione (5)}

A solution of $3(500 \mathrm{mg}, 1.31 \mathrm{mmol}), N$-bromosuccinimide $(700 \mathrm{mg}, 3.93 \mathrm{mmol})$ and benzoyl peroxide $(10 \mathrm{mg}, 0.04 \mathrm{mmol})$ in $\mathrm{CCl}_{4}(40 \mathrm{ml})$ was heated at reflux for 2.5 hours. To the mixture was added water $(40 \mathrm{ml})$ and the product was extracted with $\mathrm{CHCl}_{3}(3 \times 40 \mathrm{ml})$. The combined organic extracts were dried $\left(\mathrm{Na}_{2} \mathrm{SO}_{4}\right)$ and evaporated. The resulting crude product was purified by column chromatography, eluting with $\mathrm{CHCl}_{3}$. A yellow crystalline solid was obtained $520 \mathrm{mg}(74 \%)$ : MP $226 \sim 227^{\circ} \mathrm{C}$; NMR Table 3; IR $\left(\mathrm{CHCl}_{3}\right) \mathrm{cm}^{-1} 2975,1730,1700,1670,1585$; MS calcd for $\mathrm{C}_{22} \mathrm{H}_{24} \mathrm{Br}_{2} \mathrm{~N}_{2} \mathrm{O}_{4}, 538.010$, observed 538.007.

\section{2,8-Dimethoxy-4,6-dipropyl-5,10-dioxo-5,10-dihydropyrido[3,2-g]quinoline-3,7-diacetonitrile (6)}

To a solution of $5(500 \mathrm{mg}, 0.93 \mathrm{mmol})$ in $\mathrm{CH}_{2} \mathrm{Cl}_{2}(50 \mathrm{ml})$ was added dropwise a solution of tetraethylammonium cyanide $(289 \mathrm{mg}, 1.85 \mathrm{mmol})$ in $\mathrm{CH}_{2} \mathrm{Cl}_{2}(50 \mathrm{ml})$ at $0^{\circ} \mathrm{C}$ in 4 hours. In a similar 
Table 3. ${ }^{1} \mathrm{H}$ NMR parameters $(\delta)$ for diazaquinomycin A analogues. ${ }^{2}$

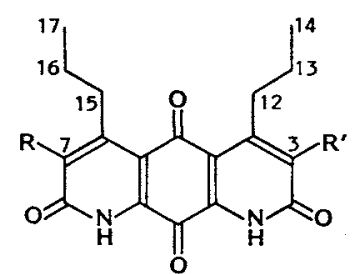

Type I

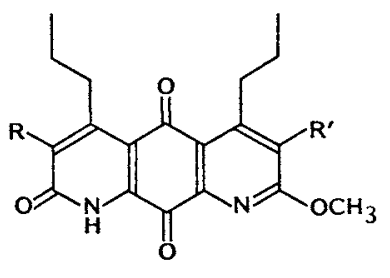

Type II

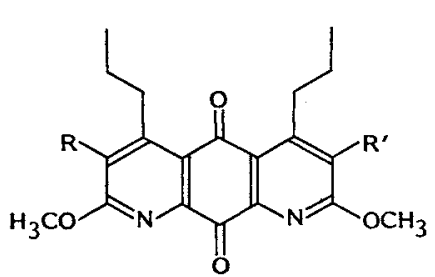

Type III

\begin{tabular}{|c|c|c|c|c|c|c|c|c|}
\hline $\begin{array}{l}\text { Com- } \\
\text { pound }\end{array}$ & Type & $\mathrm{R}$ & $\mathbf{R}^{\prime}$ & $\begin{array}{l}12-\mathrm{H} \\
15-\mathrm{H}\end{array}$ & $\begin{array}{l}13-\mathrm{H}, \\
16-\mathrm{H}\end{array}$ & $\begin{array}{l}14-\mathrm{H}, \\
17-\mathrm{H}\end{array}$ & $\mathrm{OCH}_{3}$ & Other \\
\hline 2 & II & $\mathrm{CH}_{3}$ & $\mathrm{CH}_{3}$ & $3.07 \mathrm{t}$ & $1.59 \mathrm{~m}$ & $1.10 \mathrm{t}$ & $4.13 \mathrm{~s}$ & $2.27 \mathrm{~s}\left(3-\mathrm{CH}_{3}\right), 2.30 \mathrm{~s}\left(7-\mathrm{CH}_{3}\right)$ \\
\hline 3 & III & $\mathrm{CH}_{3}$ & $\mathrm{CH}_{3}$ & $3.09 t$ & $1.57 \mathrm{~m}$ & $1.09 t$ & $4.15 \mathrm{~s}$ & $2.28 \mathrm{~s}\left(3,7-\mathrm{CH}_{3}\right)$ \\
\hline 4 & II & $\mathrm{CH}_{3}$ & $\mathrm{CH}_{2} \mathrm{Br}$ & $2.69 t$ & $1.59 \mathrm{~m}$ & $1.17 \mathrm{t}$ & $4.14 \mathrm{~s}$ & $2.31 \mathrm{~s}\left(7-\mathrm{CH}_{3}\right), 4.60 \mathrm{~s}\left(3-\mathrm{CH}_{2}\right)$ \\
\hline 5 & III & $\mathrm{CH}_{2} \mathrm{Br}$ & $\mathrm{CH}_{2} \mathrm{Br}$ & $3.20 t$ & $1.69 \mathrm{~m}$ & $1.16 \mathrm{t}$ & $4.22 \mathrm{~s}$ & $4.62 \mathrm{~s}\left(3,7-\mathrm{CH}_{2}\right)$ \\
\hline 6 & III & $\mathrm{CH}_{2} \mathrm{CN}$ & $\mathrm{CH}_{2} \mathrm{CN}$ & $3.17 \mathrm{t}$ & $1.64 \mathrm{~m}$ & $1.15 \mathrm{t}$ & $4.24 \mathrm{~s}$ & $3.82 \mathrm{~s}\left(3,7-\mathrm{CH}_{2}\right)$ \\
\hline 7 & $\mathbf{I}$ & $\mathrm{CH}_{2} \mathrm{COOEt}$ & $\mathrm{CH}_{2} \mathrm{COOEt}$ & $3.00 \mathrm{t}$ & $1.54 \mathrm{~m}$ & $1.08 t$ & & $\begin{array}{l}1.27 \mathrm{t}\left(\mathrm{COOCH}_{2} \mathrm{CH}_{3}\right), 3.78 \mathrm{~s}\left(3,7-\mathrm{CH}_{2}\right) \\
4.19 \mathrm{q}\left(\mathrm{COOCH}_{2} \mathrm{CH}_{3}\right)\end{array}$ \\
\hline 8 & $\mathbf{I}$ & $\mathrm{CH}_{2} \mathrm{COOH}$ & $\mathrm{CH}_{2} \mathrm{COOH}$ & $2.96 t$ & $1.30 \mathrm{~m}$ & $1.00 \mathrm{t}$ & & $3.60 \mathrm{~s}\left(3,7-\mathrm{CH}_{2}\right)$ \\
\hline 9 & III & $\mathrm{CH}_{2} \mathrm{CH}(\mathrm{COOEt})_{2}$ & $\mathrm{CH}_{2} \mathrm{CH}(\mathrm{COOE} t)_{2}$ & $3.15 \mathrm{t}$ & $1.55 \mathrm{~m}$ & $1.08 t$ & $4.15 \mathrm{~s}$ & $\begin{array}{l}1.21 \mathrm{t}\left(\mathrm{CH}\left(\mathrm{COOCH}_{2} \mathrm{CH}_{3}\right)_{2}\right), 3.34 \mathrm{~d}\left(3,7-\mathrm{CH}_{2}\right) \text {, } \\
3.38 \mathrm{t}\left(\mathrm{CH}\left(\mathrm{COOCH}_{2} \mathrm{CH}_{3}\right)_{2}\right), \\
4.15 \mathrm{q}\left(\mathrm{CH}\left(\mathrm{COOCH}_{2} \mathrm{CH}_{3}\right)_{2}\right)\end{array}$ \\
\hline 10 & $\mathbf{I}$ & $\mathrm{CH}_{2} \mathrm{CH}(\mathrm{COOEt})_{2}$ & $\mathrm{CH}_{2} \mathrm{CH}(\mathrm{COOEt})_{2}$ & $3.07 \mathrm{t}$ & $1.50 \mathrm{~m}$ & $1.16 t$ & & $\begin{array}{l}1.24 \mathrm{t}\left(\mathrm{CH}\left(\mathrm{COOCH}_{2} \mathrm{CH}_{3}\right)_{2}\right), 3.29 \mathrm{~d}\left(3,7-\mathrm{CH}_{2}\right) \text {, } \\
4.18 \mathrm{q}\left(\mathrm{CH}\left(\mathrm{COOCH}_{2} \mathrm{CH}_{3}\right)_{2}\right.\end{array}$ \\
\hline
\end{tabular}




\begin{tabular}{|c|c|c|c|c|c|c|c|c|}
\hline 12 & III & $\mathrm{CH}_{2} \mathrm{CH}(\mathrm{COOH})_{2}$ & $\mathrm{CH}_{2} \mathrm{CH}(\mathrm{COOH})_{2}$ & $3.28 \mathrm{t}$ & $1.50 \mathrm{~m}$ & $1.09 \mathrm{t}$ & $4.14 \mathrm{~s}$ & $3.40 \mathrm{~d}\left(3,7-\mathrm{CH}_{2}\right), 3.70 \mathrm{t}\left(\mathrm{CH}(\mathrm{COOH})_{2}\right)$ \\
\hline 13 & III & $\mathrm{CH}_{2} \mathrm{CH}_{2} \mathrm{COOH}$ & $\mathrm{CH}_{2} \mathrm{CH}_{2} \mathrm{COOH}$ & $3.08 \mathrm{t}$ & $1.55 \mathrm{~m}$ & $1.05 \mathrm{t}$ & $4.16 \mathrm{~s}$ & $2.60 \mathrm{t}\left(\mathrm{CH}_{2} \mathrm{COOH}\right), 3.08 \mathrm{t}\left(3,7-\mathrm{CH}_{2}\right)$ \\
\hline 14 & $\mathbf{I}$ & $\mathrm{CH}_{2} \mathrm{CH}_{2} \mathrm{COOEt}$ & $\mathrm{CH}_{2} \mathrm{CH}_{2} \mathrm{COOEt}$ & $3.06 \mathrm{t}$ & $1.51 \mathrm{~m}$ & $1.11 \mathrm{t}$ & & $1.27 \mathrm{t}\left(\mathrm{CH}_{2} \mathrm{COOCH}_{2} \mathrm{CH}_{3}\right)$ \\
\hline & & & & & & & & $\begin{array}{l}2.61 \mathrm{t}\left(\mathrm{CH}_{2} \mathrm{COOCH}_{2} \mathrm{CH}_{3}\right) \text {, } \\
3.06 \mathrm{t}\left(3,7-\mathrm{CH}_{2}\right), 4.15 \mathrm{q}\left(\mathrm{CH}_{2} \mathrm{COOCH}_{2} \mathrm{CH}_{3}\right)\end{array}$ \\
\hline 16 & III & $\mathrm{CH}_{2} \mathrm{OH}$ & $\mathrm{CH}_{2} \mathrm{OH}$ & $3.13 \mathrm{t}$ & $1.61 \mathrm{~m}$ & $1.08 \mathrm{t}$ & $4.18 \mathrm{~s}$ & $4.79 \mathrm{~s}\left(3,7-\mathrm{CH}_{2}\right)$ \\
\hline 17 & UII & $\mathrm{COOH}$ & $\mathrm{COOH}$ & $3.11 \mathrm{t}$ & $1.65 \mathrm{~m}$ & $1.06 \mathrm{t}$ & $4.16 \mathrm{~s}$ & \\
\hline 18 & I & COOEt & COOEt & $2.91 \mathrm{t}$ & $1.60 \mathrm{~m}$ & $1.06 \mathrm{t}^{\prime}$ & & $1.20 \mathrm{t}\left(\mathrm{COOCH}_{2} \mathrm{CH}_{3}\right), 4.40 \mathrm{q}\left(\mathrm{COOCH}_{2} \mathrm{CH}_{3}\right)$ \\
\hline 20 & II & $\mathrm{CH}_{3}$ & $\mathrm{CH}_{2} \mathrm{OAc}$ & $3.10 \mathrm{t}$ & $1.59 \mathrm{~m}$ & $1.10 \mathrm{t}$ & $4.14 \mathrm{~s}$ & $2.09 \mathrm{~s}\left(7-\mathrm{CH}_{3}\right), 2.29 \mathrm{~s}\left(\mathrm{COCH}_{3}\right), 5.23 \mathrm{~s}\left(3-\mathrm{CH}_{2}\right)$ \\
\hline 21 & 1 & $\mathrm{CH}_{3}$ & $\mathrm{CH}_{2} \mathrm{OAc}$ & $3.02 \mathrm{t}$ & $1.57 \mathrm{~m}$ & $1.10 \mathrm{t}$ & & $1.26 \mathrm{t}\left(\mathrm{COCH}_{3}\right), 2.29 \mathrm{~s}\left(7-\mathrm{CH}_{3}\right), 5.21 \mathrm{~s}\left(3-\mathrm{CH}_{2}\right)$ \\
\hline 22 & III & $\mathrm{CH}_{2} \mathrm{OAc}$ & $\mathrm{CH}_{2} \mathrm{OAc}$ & $3.17 t$ & $1.55 \mathrm{~m}$ & $1.11 \mathrm{t}$ & $4.24 \mathrm{~s}$ & $2.10 \mathrm{~s}\left(\mathrm{COCH}_{3}\right), 5.28 \mathrm{~s}\left(3,7-\mathrm{CH}_{2}\right)$ \\
\hline 23 & I & $\mathrm{CH}_{2} \mathrm{OAc}$ & $\mathrm{CH}_{2} \mathrm{OAc}$ & $3.11 \mathrm{t}$ & $1.60 \mathrm{~m}$ & $1.17 \mathrm{t}$ & & $2.08 \mathrm{~s}\left(\mathrm{COCH}_{3}\right), 5.22 \mathrm{~s}\left(3,7-\mathrm{CH}_{2}\right)$ \\
\hline 24 & I & $\mathrm{CH}_{3}$ & $\mathrm{CH}_{2} \mathrm{OEt}$ & $3.15 t$ & $1.60 \mathrm{~m}$ & $1.11 t$ & & $\begin{array}{l}1.25 \mathrm{t}\left(\mathrm{OCH}_{2} \mathrm{CH}_{3}\right), 2.28 \mathrm{~s}\left(7-\mathrm{CH}_{3}\right) \\
3.62 \mathrm{q}\left(\mathrm{OCH}_{2} \mathrm{CH}_{3}\right), 4.58 \mathrm{~s}\left(3-\mathrm{CH}_{2}\right)\end{array}$ \\
\hline 25 & I & $\mathrm{CH}_{2} \mathrm{OEt}$ & $\mathrm{CH}_{2} \mathrm{OEt}$ & $3.15 \mathrm{t}$ & $1.65 \mathrm{~m}$ & $1.10 \mathrm{t}$ & & $\begin{array}{l}1.24 \mathrm{t}\left(\mathrm{OCH}_{2} \mathrm{CH}_{3}\right), 3.63 \mathrm{q}\left(\mathrm{OCH}_{2} \mathrm{CH}_{3}\right), \\
4.95 \mathrm{~s}\left(3,7-\mathrm{CH}_{2}\right)\end{array}$ \\
\hline 26 & III & & & $3.34 \mathrm{t}$ & $1.59 \mathrm{~m}$ & $1.11 \mathrm{t}$ & $4.13 \mathrm{~s}$ & $\begin{array}{l}2.48 \mathrm{t}\left(\mathrm{NCH}_{2} \mathrm{CH}_{2} \mathrm{O}\right), 3.60 \mathrm{~s}\left(3,7-\mathrm{CH}_{2}\right) \\
3.65 \mathrm{t}\left(\mathrm{NCH}_{2} \mathrm{CH}_{2} \mathrm{O}\right)\end{array}$ \\
\hline 27 & I & $\mathrm{CH}_{2}$ & $\mathrm{CH}_{2} \mathrm{~N}$ & $3.18 \mathrm{t}$ & $1.75 \mathrm{~m}$ & $1.10 \mathrm{t}$ & & $\begin{array}{l}2.55 \mathrm{t}\left(\mathrm{NCH}_{2} \mathrm{CH}_{2} \mathrm{O}\right), 3.65 \mathrm{~s}\left(3,7-\mathrm{CH}_{2}\right) \\
3.70 \mathrm{t}\left(\mathrm{NCH}_{2} \mathrm{CH}_{2} \mathrm{O}\right)\end{array}$ \\
\hline
\end{tabular}

Field strengths are expressed in units of $\delta(\mathrm{ppm})$. 
manner to the preparation of $\mathbf{5}$, the mixture was treated and purified to give a yellow crystalline solid, $306 \mathrm{mg}(87 \%)$ : MP $190 \sim 193^{\circ} \mathrm{C}$; NMR Table 3; IR $\left(\mathrm{CHCl}_{3}\right) \mathrm{cm}^{-1} 2955,1700,1665,1585$; MS calcd for $\mathrm{C}_{24} \mathrm{H}_{24} \mathrm{~N}_{4} \mathrm{O}_{4} 432.180$, observed 432.180 .

\section{4,6-Dipropyl-2,5,8,10-tetraoxo-1,2,5,8,9,10-hexahydropyrido[3,2-g]quinoline-3,7-diethyl Diacetate} $\underline{(7)}$

A solution of $6(52 \mathrm{mg}, 0.12 \mathrm{mmol})$ in $30 \% \mathrm{H}_{2} \mathrm{SO}_{4}$ - EtOH $(6 \mathrm{ml})$ was heated at reflux for 2 hours. To the mixture was added water $(5 \mathrm{ml})$ and the product was extracted with $\mathrm{CHCl}_{3}(3 \times 5 \mathrm{ml})$. The combined organic extracts were dried $\left(\mathrm{Na}_{2} \mathrm{SO}_{4}\right)$ and evaporated. The resulting crude product was purified by column chromatography on silica gel, eluting with $\mathrm{CHCl}_{3}-\mathrm{MeOH}(50: 1)$. A red crystalline solid was obtained, $33 \mathrm{mg}(55 \%)$ : MP $267 \sim 268^{\circ} \mathrm{C}$; NMR Table 3; IR $\left(\mathrm{CHCl}_{3}\right) \mathrm{cm}^{-1} 3360$, 1735, 1660; MS calcd for $\mathrm{C}_{28} \mathrm{H}_{30} \mathrm{~N}_{2} \mathrm{O}_{8}, 498.200$, observed 498.201.

4,6-Dipropyl-2,5,8,10-tetraoxo-1,2,5,8,9,10-hexahydropyrido[3,2-g]quinoline-3,7-diacetic Acid (8)

To a solution of $7(30 \mathrm{mg}, 0.06 \mathrm{mmol})$ in $80 \%$ EtOH $-\mathrm{H}_{2} \mathrm{O}(12 \mathrm{ml})$ was added $\mathrm{NaOH}(30 \mathrm{mg}$, $0.75 \mathrm{mmol}$ ) and the mixture was stirred at $50^{\circ} \mathrm{C}$ for 30 minutes. After the mixture was acidified with $1 \mathrm{~N} \mathrm{HCl}$, the precipitate was filtered and the solid was washed with water $(12 \mathrm{ml})$. This solid was dried in vacuo to give a red crystalline solid, $21 \mathrm{mg}(82 \%)$ : MP $300^{\circ} \mathrm{C}$; NMR Table $3 ; \mathrm{IR}\left(\mathrm{CHCl}_{3}\right)$ $\mathrm{cm}^{-1} 3600 \sim 2500,2975,2945,2880,1710,1645$; field desorption (FD)-MS $m / z 443\left(\mathrm{M}^{+}+1\right)$.

Anal Calcd for $\mathrm{C}_{22} \mathrm{H}_{22} \mathrm{~N}_{2} \mathrm{O}_{8}$ : C 59.73, H 4.98, N 6.33.

$$
\text { Found: } \quad \mathrm{C} 59.55, \mathrm{H} 4.78, \mathrm{~N} 6.03 \text {. }
$$

2,8-Dimethoxy-3,7-dimethyl-4,6-dipropyl-5,10-dioxo-5,10-dihydropyrido[3,2-g]quinoline- $3^{\prime}, 7^{\prime}-$ dimalonic Acid Tetraethyl Ester (9)

To a suspension of sodium hydride $(60 \%$ in oil) $(388 \mathrm{mg}, 16.2 \mathrm{mmol})$ in dry THF (100 ml) was added dropwise diethyl malonate $(1.8 \mathrm{ml}, 11.8 \mathrm{mmol})$, and the reaction mixture was stirred for 0.5 hour to prepare the anion of diethyl malonate. To the mixture was added a solution of 5 ( $1.5 \mathrm{~g}$, $2.79 \mathrm{mmol})$ in dry THF $(60 \mathrm{ml})$, and the resulting solution was stirred for 0.5 hour at room temperature. To the mixture was added $\mathrm{EtOH}(0.5 \mathrm{ml})$ to quench the reaction. After concentration in vacuo, water $(60 \mathrm{ml})$ was added to the residue. The mixture was acidified with $1 \mathrm{~N} \mathrm{HCl}$, and extracted with $\mathrm{CHCl}_{3}(3 \times 60 \mathrm{ml})$. The combined extracts were dxied $\left(\mathrm{Na}_{2} \mathrm{SO}_{4}\right)$ and concentrated in vacuo. The resulting crude product was purified by column chromatography on silica gel, eluting with $\mathrm{CHCl}_{3}$. A yellow crystalline solid was obtained, $1.3 \mathrm{~g}(74 \%): \mathrm{MP} 97 \sim 100^{\circ} \mathrm{C}$; NMR Table 3; IR $\left(\mathrm{CHCl}_{3}\right)$ $\mathrm{cm}^{-1} 3010,1740,1705,1675,1595$; MS calcd for $\mathrm{C}_{36} \mathrm{H}_{46} \mathrm{~N}_{2} \mathrm{O}_{12}, 698.305$, observed 698.307.

3,7-Dimethyl-4,6-dipropyl-2,5,8,10-tetraoxo-1,2,5,8,9,10-hexahydropyrido[3,2-g]quinoline- $3^{\prime}, 7^{\prime}-$ dimalonic Acid Tetraethyl Ester (10)

A solution of $9(100 \mathrm{mg}, 0.14 \mathrm{mmol})$ in $30 \% \mathrm{H}_{2} \mathrm{SO}_{4}-\mathrm{EtOH}(30 \mathrm{ml})$ was heated at reflux for 12 hours. The mixture was cooled, and concentrated in vacuo. To the mixture was added water $(5 \mathrm{ml})$, and the product was extracted with $\mathrm{CHCl}_{3}$. The organic phase was dried $\left(\mathrm{Na}_{2} \mathrm{SO}_{4}\right)$ and concentrated in vacuo. The resulting crude product was purified by column chromatography on silica gel, eluting with $\mathrm{CHCl}_{3}-\mathrm{MeOH}(10: 1)$. A red crystalline solid was obtained, $91 \mathrm{mg}(95 \%)$ : $\mathrm{MP} 89 \sim 92^{\circ} \mathrm{C}$; NMR Table 3; IR $\left(\mathrm{CHCl}_{3}\right) \mathrm{cm}^{-1} 3350,2975,1725,1655$; MS calcd for $\mathrm{C}_{34} \mathrm{H}_{42} \mathrm{~N}_{2} \mathrm{O}_{12}, 670.274$, observed 670.274 .

2,8-Dimethoxy-3,7-dimethyl-4,6-dipropyl-5,10-dioxo-5,10-dihydropyrido[3,2-g]quinoline- $3^{\prime}, 7^{\prime}-$ dimalonic Acid (12)

A mixture of $9(50 \mathrm{mg}, 0.07 \mathrm{mmol})$ in $80 \% \mathrm{EtOH}-\mathrm{H}_{2} \mathrm{O}(15 \mathrm{ml})$ was treated with $\mathrm{NaOH}(30 \mathrm{mg}$, $0.75 \mathrm{mmol}$ ) and the mixture was heated at reflux for 1 hour. The mixture was acidified with $1 \mathrm{~N}$ $\mathrm{HCl}$, and $\mathrm{H}_{2} \mathrm{O}(15 \mathrm{ml})$ was added. After extracting with $\mathrm{CHCl}_{3}$, the organic phase was dried $\left(\mathrm{Na}_{2} \mathrm{SO}_{4}\right)$ and concentrated in vacuo. A yellow crystalline solid was obtained $34 \mathrm{mg}(82 \%)$ : MP $300^{\circ} \mathrm{C}$; NMR Table 3; IR (KBr) cm $\mathrm{cm}^{-1} 3700 \sim 2500,2975,1710,1670,1585$; FD-MS $m / z 587\left(\mathrm{M}^{+}+1\right)$.

Anal Calcd for $\mathrm{C}_{28} \mathrm{H}_{30} \mathrm{~N}_{2} \mathrm{O}_{12}$ : C 57.34, H 5.12, N 4.78.

Found:

C 57.18, H 5.03, N 5.01 . 
2,8-Dimethoxy-4,6-dipropyl-5,10-dioxo-5,10-dihydropyrido[3,2-g]quinoline-3,7-propionic Acid (13)

A solution of $12(50 \mathrm{mg}, 0.09 \mathrm{mmol})$ in pyridine $(10 \mathrm{ml})$ was heated at reflux for 4 hours. The mixture was concentrated in vacuo, and acidified with $1 \mathrm{~N} \mathrm{HCl}$. After extracting with $\mathrm{CHCl}_{3}$, the organic phase was dried $\left(\mathrm{Na}_{2} \mathrm{SO}_{4}\right)$ and concentrated in vacuo. The resulting crude product was purified by column chromatography on silica gel, eluting with $\mathrm{CHCl}_{3}$. A yellow crystalline solid was obtained, $34 \mathrm{mg}(94 \%)$ : MP 253 255 ${ }^{\circ} \mathrm{C}$; NMR Table 3; IR $\left(\mathrm{CHCl}_{3}\right) \mathrm{cm}^{-1} 3600 \sim 2500,2960,1705,1665,1585$; MS calcd for $\mathrm{C}_{26} \mathrm{H}_{30} \mathrm{~N}_{2} \mathrm{O}_{3}, 498.200$, observed 498.201.

4,6-Dipropyl-2,5,8,10-tetraoxo-1,2,5,8,9,10-hexahydropyrido[3,2-g]quinoline-3,7-dipropionic Acid Diethyl Ester (14)

A solution of $13(34 \mathrm{mg}, 0.07 \mathrm{mmol})$ in $30 \% \mathrm{H}_{2} \mathrm{SO}_{4}$ - EtOH $(15 \mathrm{ml})$ was heated at reflux for 4 hours. After the reaction was complete according to TLC analysis, in a similar manner to the preparation of 5, the mixture was treated and purified to give a red crystalline solid, $19 \mathrm{mg}(53 \%)$ : MP $175 \sim 177^{\circ} \mathrm{C}$; NMR Table 3; IR $\left(\mathrm{CHCl}_{3}\right) \mathrm{cm}^{-1} 3355,2975,1730,1650$; MS calcd for $\mathrm{C}_{28} \mathrm{H}_{34} \mathrm{~N}_{2} \mathrm{O}_{8}$, 526.232, observed 526.232.

\section{3,7-Dihydroxymethyl-2,8-dimethoxy-4,6-dipropylpyrido[3,2-g]quinoline-5,10-dione (16)}

A solution of $5(80 \mathrm{mg}, 0.15 \mathrm{mmol})$ in $33 \%$ THF $-\mathrm{H}_{2} \mathrm{O}(15 \mathrm{ml})$ was heated at reflux for 2 days. The mixture was concentrated in vacuo, and water $(30 \mathrm{ml})$ was added. After extracting with $\mathrm{CHCl}_{3}$, the organic phase was dried $\left(\mathrm{Na}_{2} \mathrm{SO}_{4}\right)$ and concentrated in vacuo. The resulting crude product was purified by column chromatography on silica gel, eluting with $\mathrm{CHCl}_{3}-\mathrm{MeOH}(20: 1)$. A yellow crystalline solid was obtained, $27 \mathrm{mg}(44 \%)$ : MP $230 \sim 235^{\circ} \mathrm{C}$; NMR Table 3; IR $\left(\mathrm{CHCl}_{3}\right) \mathrm{cm}^{-1} 3400$, $2955,1700,1670,1585$; MS calcd for $\mathrm{C}_{22} \mathrm{H}_{28} \mathrm{~N}_{2} \mathrm{O}_{6}, 414.179$, observed 414.179.

2,8-Dimethoxy-4,6-dipropyl-5,10-dioxo-5,10-dihydropyrido[3,2-g]quinoline-3,7-dicarboxylic Acid (17)

To a solution of $16(550 \mathrm{mg}, 1.33 \mathrm{mmol})$ in acetone $(50 \mathrm{ml})$ was added a solution of $\mathrm{CrO}_{3}-\mathrm{H}_{2} \mathrm{SO}_{4}$ in acetone and the resulting mixture was stirred at $0^{\circ} \mathrm{C}$ for 10 minutes. To the mixture was added 2-propanol and the mixture was concentrated in vacuo. Further water $(150 \mathrm{ml})$ was added and the product was extracted with $\mathrm{CHCl}_{3}$. The organic phase was dried $\left(\mathrm{Na}_{2} \mathrm{SO}_{4}\right)$, and concentrated in vacuo. A yellow crystalline solid was obtained, $510 \mathrm{mg}(87 \%)$ : MP $231 \sim 235^{\circ} \mathrm{C}$; NMR Table 3 ; IR $\left(\mathrm{CHCl}_{3}\right) \mathrm{cm}^{-1} 3600 \sim 2500,2980,1745,1710,1670,1585$; MS calcd for $\mathrm{C}_{22} \mathrm{H}_{22} \mathrm{~N}_{2} \mathrm{O}_{8}, 442.138$, observed 442.138 .

4,6-Dipropyl-2,5,8,10-tetraoxo-1,2,5,8,9,10-hexahydropyrido[3,2-g]quinoline-3,7-dicarboxylic Acid Diethyl Ester (18)

A solution of $17(210 \mathrm{mg}, 0.48 \mathrm{mmol})$ in $30 \% \mathrm{H}_{2} \mathrm{SO}_{4}-\mathrm{EtOH}(30 \mathrm{ml})$ was heated at reflux for 5 hours. The mixture was concentrated in vacuo, and water $(20 \mathrm{ml})$ was added. After extracting with $\mathrm{CHCl}_{3}$, the organic phase was dried $\left(\mathrm{Na}_{2} \mathrm{SO}_{4}\right)$ and concentrated in vacuo. The resulting crude product was purified by column chromatography on silica gel, eluting with $\mathrm{CHCl}_{3}-\mathrm{MeOH}(25: 1)$. A red crystalline solid was obtained, $53 \mathrm{mg}(50 \%)$ : MP $230 \sim 233^{\circ} \mathrm{C}$; NMR Table 3; IR $\left(\mathrm{CHCl}_{3}\right) \mathrm{cm}^{-1} 3350$, $2975,1735,1650 ;$ MS calcd for $\mathrm{C}_{24} \mathrm{H}_{26} \mathrm{~N}_{2} \mathrm{O}_{8}, 470.169$, observed 470.172 .

\section{3-Acetoxymethyl-2-methoxy-7-methyl-4,6-dipropylpyrido[3,2-g]quinoline-5,8,10(9H)-trione (20)}

To a solution of $4(114 \mathrm{mg}, 0.26 \mathrm{mmol})$ in acetic acid $(10 \mathrm{ml})$ was added $\mathrm{NaOH}(30 \mathrm{mg}, 0.75 \mathrm{mmol})$ and the resulting mixture was stirred at $100^{\circ} \mathrm{C}$ for 5 hours. After concentration in vacuo, to the residue was added water $(10 \mathrm{ml})$ and the product was extracted with $\mathrm{CHCl}_{3}$. The organic phase was dried $\left(\mathrm{Na}_{2} \mathrm{SO}_{4}\right)$ and concentrated in vacuo. The resulting crude product was purified by column chromatography on silica gel, eluting with $\mathrm{CHCl}_{3}-\mathrm{MeOH}(25: 1)$. An orange crystalline solid was obtained, $104 \mathrm{mg}$ (96\%): MP 188 190 ${ }^{\circ}$; NMR Table 3; IR $\left(\mathrm{CHCl}_{3}\right) \mathrm{cm}^{-1} 3350,2945,1740,1655$, 1575; MS calcd for $\mathrm{C}_{23} \mathrm{H}_{26} \mathrm{~N}_{2} \mathrm{O}_{6}, 426.179$, observed 426.179.

3-Acetoxymethyl-7-methyl-4,6-dipropylpyrido[3,2-g]quinoline-2,5,8,10(1 H,9H)-tetrone (21)

To a solution of 20 in acetic acid $(10 \mathrm{ml})$ was added conc $\mathrm{H}_{2} \mathrm{SO}_{4}(1 \mathrm{ml})$, and the resulting solution 
was heated at reflux for 5 hours. To the mixture was added water $(10 \mathrm{ml})$, and the product was extracted with $\mathrm{CHCl}_{3}$. After extraction, in a similar manner to the preparation of $\mathbf{5}$, the mixture was treated and purified to give a red crystalline solid, $65 \mathrm{mg}(65 \%)$ : MP $271 \sim 272^{\circ} \mathrm{C}$; NMR Table 3; IR $\left(\mathrm{CHCl}_{3}\right) \mathrm{cm}^{-1} 3350,2945,1740,1655$; MS calcd for $\mathrm{C}_{22} \mathrm{H}_{24} \mathrm{~N}_{2} \mathrm{O}_{6}, 412.164$, observed 412.163.

\section{3,7-Diacetoxymethyl-2,8-dimethoxy-4,6-dipropylpyrido[3,2-g]quinoline-5,10-dione (22)}

To a solution of $5(50 \mathrm{mg}, 0.09 \mathrm{mmol})$ in acetic acid $(6 \mathrm{ml})$ was added $\mathrm{NaOH}(30 \mathrm{mg}, 0.75 \mathrm{mmol})$ and the resulting mixture was stirred at $100^{\circ} \mathrm{C}$ for 3 hours. After the reaction was complete according to TLC analysis, in a similar manner to the preparation of $\mathbf{5}$, the mixture was treated and purified to give a yellow crystalline solid, $42 \mathrm{mg}(91 \%)$ : MP $98 \sim 100^{\circ} \mathrm{C}$; NMR Table 3; IR $\left(\mathrm{CHCl}_{3}\right) \mathrm{cm}^{-1} 2945$, 1740, 1660, 1575; FD-MS $m / z 499\left(\mathrm{M}^{+}+1\right)$.

$\begin{array}{ll}\text { Anal Calcd for } \mathrm{C}_{26} \mathrm{H}_{30} \mathrm{~N}_{2} \mathrm{O}_{8}: & \mathrm{C} 62.65, \mathrm{H} 6.02, \mathrm{~N} 5.62 . \\ \text { Found: } & \text { C 62.78, H 6.15, N 5.49. }\end{array}$

3,7-Diacetoxymethyl-4,6-dipropylpyrido[3,2-g]quinoline-2,5,8,10(1H,9H)-tetrone (23)

To a solution of $22(42 \mathrm{mg}, 0.09 \mathrm{mmol})$ in acetic acid (4 ml) was added conc $\mathrm{H}_{2} \mathrm{SO}_{4}(0.2 \mathrm{ml})$, and the resulting solution was heated at reflux for 3 hours. After the reaction was complete according to TLC analysis, in a similar manner to the preparation of 11, the mixture was treated and purified to give a red crystalline solid, $25 \mathrm{mg}(63 \%)$ : MP $165 \sim 168^{\circ} \mathrm{C}$; NMR Table 3; IR $\left(\mathrm{CHCl}_{3}\right) \mathrm{cm}^{-1} 3350$, $2945,1740,1665$; MS calcd for $\mathrm{C}_{24} \mathrm{H}_{28} \mathrm{~N}_{2} \mathrm{O}_{8}, 470.169$, observed 470.169 .

3-Ethoxymethyl-7-methyl-4,6-dipropylpyrido[3,2-g]quinoline-2,5,8,10(1H,9H)-tetrone (24)

A solution of $4(320 \mathrm{mg}, 0.72 \mathrm{mmol})$ in $30 \% \mathrm{H}_{2} \mathrm{SO}_{4}-\mathrm{EtOH}(120 \mathrm{ml})$ was heated at reflux for 10 hours. After the reaction was complete according to TLC analysis, in a similar manner to the preparation of 5 , the mixture was treated and purified to give a red crystalline solid, $100 \mathrm{mg}(35 \%)$ : MP 286 288 ${ }^{\circ} \mathrm{C}$; NMR Table 3; IR $\left(\mathrm{CHCl}_{3}\right) \mathrm{cm}^{-1} 2975,1670,1630$; MS calcd for $\mathrm{C}_{22} \mathrm{H}_{26} \mathrm{~N}_{2} \mathrm{O}_{5}$, 398.184, observed 398.183 .

\section{3,7-Diethoxymethyl-4,6-dipropylpyrido[3,2-g]quinoline-2,5,8,10(1H,9H)-tetrone (25)}

To a solution of $5(52 \mathrm{mg}, 0.09 \mathrm{mmol})$ in $30 \% \mathrm{H}_{2} \mathrm{SO}_{4}$ - EtOH $(8 \mathrm{ml})$ was heated at reflux for 10 hours. After the reaction was complete according to TLC analysis, in a similar manner to the preparation of 5 , the mixture was treated and purified to give a red crystalline solid, $13 \mathrm{mg}(32 \%)$ : MP $233 \sim 235^{\circ} \mathrm{C}$; NMR Table 3; IR $\left(\mathrm{CHCl}_{3}\right) \mathrm{cm}^{-1} 3350,2980,1655,1630$; MS calcd for $\mathrm{C}_{24} \mathrm{H}_{30} \mathrm{~N}_{2} \mathrm{O}_{6}$, 442.211, observed 442.211 .

\section{2,8-Dimethoxy-3,7-dimorpholinomethyl-4,6-dipropylpyrido[3,2-g]quinoline-5,10-dione (26)}

A solution of $5(52 \mathrm{mg}, 0.09 \mathrm{mmol})$ in morpholine $(2 \mathrm{ml}, 22.9 \mathrm{mmol})$ was stirred for 2 hours at room temperature. The mixture was concentrated to dryness in vacuo and to the residue was added $1 \mathrm{~N} \mathrm{Na}_{2} \mathrm{CO}_{3}(10 \mathrm{ml})$. After extracting with $\mathrm{CHCl}_{3}$, the organic phase was dried $\left(\mathrm{Na}_{2} \mathrm{SO}_{4}\right)$ and the solvent removed in vacuo. The resulting crude product was purified by column chromatography on silica gel, eluting with $\mathrm{CHCl}_{3}-\mathrm{MeOH}(40: 1)$. A yellow crystalline solid was obtained, $33.3 \mathrm{mg}$ $(64 \%)$ : MP 94 95 ${ }^{\circ}$; NMR Table 3; IR $\left(\mathrm{CHCl}_{3}\right) \mathrm{cm}^{-1} 2975,1700,1670,1590$; MS calcd for $\mathrm{C}_{30} \mathrm{H}_{40} \mathrm{~N}_{4} \mathrm{O}_{8}, 552.295$, observed 552.293.

\section{3,7-Dimorpholinomethyl-4,6-dipropylpyrido[3,2-g]quinoline-2,5,8,10(1 H,9H)-tetrone (27)}

A solution of $26(33.3 \mathrm{mg}, 0.06 \mathrm{mmol})$ in $30 \% \mathrm{H}_{2} \mathrm{SO}_{4}-\mathrm{EtOH}(30 \mathrm{ml})$ was stirred at $100^{\circ} \mathrm{C}$ for 1.5 hours. After the reaction was complete according to TLC analysis, in a similar manner to the preparation of 10, the mixture was treated and purified to give a red crystalline solid, $22.1 \mathrm{mg}(67 \%)$ : MP 210 212 ${ }^{\circ} \mathrm{C}$; NMR Table 3; IR $\left(\mathrm{CHCl}_{3}\right) \mathrm{cm}^{-1} 2975$, 1660; FD-MS $m / z 525\left(\mathrm{M}^{+}+1\right)$.

Anal Calcd for $\mathrm{C}_{28} \mathrm{H}_{38} \mathrm{~N}_{4} \mathrm{O}_{6}$ : C 64.12, H 6.87, N 10.69. Found: C $63.97, \mathrm{H} 6.69, \mathrm{~N} 10.82$.

Antimicrobial Activity and Reversal Test with TdR

MIC against E. faecium IFO 3181 was assayed by agar dilution method with Folic Acid Assay Medium "Nissui" containing $1.0 \mathrm{ng} / \mathrm{ml}$ of pteroic acid at $37^{\circ} \mathrm{C} . .^{2} \quad$ Reversal of antimicrobial activity 
with $T d R$ was tested by conventional counter diffusion method. A compound was dissolved in DMSO at the final concentration of $0.1 \mu \mathrm{g} / \mathrm{ml}$. The solution, $50 \mu \mathrm{l}$, was applied to a sterile paper disk (i.d. $8 \mathrm{~mm}$ ) and the dried disk was placed onto the medium.

\section{HeLa Cell Culture}

A culture of HeLa cells was grown and maintained in EAGLE's minimum essential medium supplemented with $5 \%$ calf serum, benzylpenicillin $(100 \mathrm{v} / \mathrm{ml})$ and streptomycin $(100 \mu \mathrm{g} / \mathrm{ml})$ as monolayer culture. Logarithmic phase cells were harvested by treating with trypsin $(0.05 \%)$ and EDTA $(0.01 \%)$ in calcium and magnesium-free phosphate buffered saline solution. After washing with the growth medium described above, cells were incubated in wells of 96-well flat bottom microplates (Corning cell wells). Each well contained $0.1 \mathrm{ml}$ of the fresh growth medium supplemented with the compounds and $5 \times 10^{3}$ cells. The compounds were dissolved in DMSO and added to the medium at the final concentration of $1 \%$ of DMSO. The control well contained DMSO at the same rate. The cells were incubated at $37^{\circ} \mathrm{C}$ in water-saturated atmosphere of $5 \% \mathrm{CO}_{2}$ in air. After incubation for 4 days, the cell growth was observed.

\section{Assay of TMP Synthase}

The enzyme preparation was obtained according to the method described by ROBERTs ${ }^{7)}$ with some modifications. The assay of TMP synthase from Ehrlich ascites carcinoma was performed as described by CALVERT et al. ${ }^{8)}$ with some modifications described previously.

\section{Antitumor Activity}

$\mathrm{CDF}_{1}$ mice were inoculated ip on day- 0 with Meth-A fibrosarcoma $\left(1 \times 10^{\circ}\right.$ cells/mouse $)$. The tumor had been maintained by ip transfer into BALB/c mice. Beginning 24 hours after tumor cell inoculation, the compounds were administered ip daily for four consecutive days. Antitumor activity was evaluated by the increased life span in the treated groups to that in the control group $(\mathrm{T} / \mathrm{C})$.

\section{Acknowledgment}

This work was supported in part by fund from the Japan Keirin Association. The authors wish to thank Sapporo Breweries Ltd., for supply of diazaquinomycin A and for evaluation of in vivo antitumor activity.

\section{References}

1) Ōmura, S.; Y. Iwai, K. Hinotozawa, H. Tanaka, Y. Takahashi \& A. NaKagawa: OM-704 A, a new antibiotic active against Gram-positive bacteria produced by Streptomyces sp. J. Antibiotics 35: 1425 1429,1982

2) Ömura, S.; M. Murata, K. Kimura, S. Matsukura, T. Nishimara \& H. Tanaka: Screening for new antifolates of microbial origin and a new antifolate AM-8402. J. Antibiotics 38: 1016 1024, 1985

3) Murata, M.; T. Mryasaka, H. Tanaka \& S. OMmura: Diazaquinomycin A, a new antifolate antibiotic, inhibits thymidylate synthase. J. Antibiotics 38: 1025 1033, 1985

4) Omura, S.; A. Nakagawa, H. Aoyama, K. Hinotozawa \& H. Sano: The structures of diazaquinomycins A and B, new antibiotic metabolites. Tetrahedron Lett. 24:3643 3646, 1983

5) ANdreades, S. \& E. W. Zahnow: Anodic cyanations of aromatic compounds. J. Am. Chem. Soc. 91: $4181 \sim 4190,1969$

6) Simchen, G. \& H. Kobler: Synthesis of nitriles with tetraalkylammonium cyanide. Synthesis 1975: $605 \sim 607,1975$

7) RoBerts, D.: An isotopic assay for thymidylate synthetase. Biochemistry 5: 3546 3548, 1966

8) Calvert, A. H.; T. R. Jones, P. J. Dady, B. Grazelakowska-Sztabert, R. M. Paine, G. A. Taylor \& K. R. HARRAP: Quinazoline antifolates with dual biochemical loci of action. Biochemical and biological studies directed towards overcoming methotrexate resistance. Eur. J. Cancer 16: 713 722, 1980 